\title{
AUTOESTIMA E QUALIDADE DE VIDA DE UMA SÉRIE DE GESTANTES ATENDIDAS EM REDE PÚBLICA DE SAÚDE*
}

Alinne Barbosa dos Santos¹, Karolynne Elena Peluti Santos', Gina Torres Rego Monteiro², Patrícia Rezende do Prado ${ }^{3}$, Thatiana Lameira Maciel Amaral ${ }^{4}$

${ }^{1}$ Discente em enfermagem. Universidade Federal do Acre. Rio Branco-AC-Brasil.

${ }^{2}$ Médica. Doutora em Ciências da Saúde. Fundação Oswaldo Cruz. Rio de Janeiro-RJ-Brasil.

${ }^{3}$ Enfermeira. Doutoranda da Universidade Federal de São Paulo. Professora da Universidade Federal do Acre. Rio Branco-AC-Brasil. ${ }^{4}$ Enfermeira. Doutoranda em Saúde Pública e Meio Ambiente. Professora da Universidade Federal do Acre. Rio Branco-AC-Brasil.

RESUMO: O objetivo foi descrever os níveis de autoestima e de qualidade de vida de gestantes atendidas na rede pública de saúde de Rio Branco, estado do Acre. Foram entrevistadas 352 gestantes da zona urbana, com idade gestacional $\geq 35$ semanas, em 2011. Sendo empregada a Escala de Autoestima de Rosenberg e o Questionário de Qualidade de Vida da Organização Mundial de Saúde. O perfil da amostra foi de 21 a 25 anos (40,9\%), solteiras $(79,3 \%)$, não-brancas $(82,9 \%)$, escolaridade ensino médio ou superior $(66,7 \%)$, renda familiar menor que dois salários mínimos (60,2\%), multigestas (59,4\%) e gravidez atual desejada (85,5\%). Menor autoestima foi apresentada pelas grávidas não-casadas e pelas de maior classificação econômica. Observou-se menor qualidade de vida, no domínio físico, entre aquelas acima de 30 anos. Assim, a presença de companheiro e a classificação econômica parecem influenciar a autoestima e a idade, a qualidade de vida, das mulheres no período gestacional. DESCRITORES: Gravidez; Autoestima; Qualidade de vida; Saúde pública.

\section{SELF-ESTEEM AND QUALITY OF LIFE IN A SERIES OF PREGNANT WOMEN ATTENDED IN A PUBLIC HEALTH NETWORK}

\begin{abstract}
This paper aims to describe the self-esteem and quality of life levels of pregnant women attended in the public health network of Rio Branco, Acre. Three hundred and fifty-two pregnant women from the urban area with $\geq 35$ weeks gestational age were interviewed in 2011 . The Rosenberg Self-Esteem Scale and the World Health Organization Quality of Life (WHOQOL) Questionnaire were used in this study. The sample profile evidenced the following information on these women: $40.9 \%$ were in the $21-25$ age range, $79.3 \%$ were single, $82.9 \%$ were nonwhite, $66.7 \%$ had secondary or higher education, $60.2 \%$ had family income lower than two minimum wages, 59.4\% were multiparous and $85.5 \%$ wanted their current pregnancy. Lower self-esteem levels were found in unmarried pregnant women and those with greater economic status. In the physical domain, lower quality of life was noted among those above 30 years of age. Thus, during pregnancy, the presence of a partner and the economic status appear to influence women's self-esteem, while age influences their quality of life.
\end{abstract} KEYWORDS: Pregnancy, Self-esteem, Quality of Life, Public Health.

\section{AUTOESTIMA Y CUALIDAD DE VIDA DE UNA SERIE DE GESTANTES ATENDIDAS EN RED PÚBLICA DE SALUD}

RESUMEN: El objetivo fue describir los niveles de autoestima y de cualidad de vida de gestantes atendidas en la red pública de salud de Rio Branco, estado de Acre. Fueron entrevistadas 352 gestantes de la zona urbana, con edad gestacional $\geq 35$ semanas, en 2011. Fue empleada la Escala de Autoestima de Rosenberg y el Cuestionario de Cualidad de Vida de la Organización Mundial de Salud. El perfil de la muestra fue de 21 a 25 años (40,9\%), solteras (79,3\%), no blancas (82,9\%), escolaridad enseñanza media o superior $(66,7)$, renta familiar menor que dos sueldos mínimos $(60,2 \%)$, multigestas $(59,4 \%)$ y gravidez actual deseada $(85,5)$. Menor autoestima fue presentada por las embarazadas no casadas y por las de mayor clasificación económica. Se observó menor cualidad de vida, en el dominio físico, entre aquellas de más de 30 años. Así, la presencia de compañero y la clasificación económica parecen influenciar la autoestima, y la edad, la cualidad de vida de las mujeres en el periodo gestacional.

DESCRIPTORES: Gravidez; Autoestima; Cualidad de vida; Salud pública.

*Artigo extraído do Trabalho de Conclusão de Curso de Enfermagem, intitulado: Estudo da autoestima e qualidade de vida de gestantes atendidas na rede pública de saúde de Rio Branco, Acre. Universidade Federal do Acre, 2013.

Autor Correspondente:

Thatiana Lameira Maciel Amaral

Universidade Federal do Acre

BR 364, Km 04 - 69920-900 - Rio Branco-AC-Brasil

E-mail: thatianalameira27@gmail.com
Recebido: $14 / 10 / 2014$ Finalizado: $27 / 03 / 2015$ 


\section{INTRODUÇÃO}

A gravidez na vida da mulher resulta em grandes transformações que influenciam diversos aspectos biopsicossociais ${ }^{(1)}$.Trata-se, portanto, de um momento único, em que cada mulher responde de maneira diferenciada ${ }^{(2)}$.

Gestar é considerado um momento especial, porém, não para todas, pois o período gravídicopuerperal é a fase de maior incidência de transtornos psíquicos $^{(3)}$. A susceptibilidade está relacionada a fatores familiares, conjugais, sociais, culturais e de personalidade, que repercutem na saúde da mãe e do bebê com inter-relação entre as mudanças e a autoestima feminina ${ }^{(3-5)}$.

Entende-se por autoestima a apreciação que o indivíduo faz de si mesmo em relação a sua autoconfiança e ao seu autorrespeito. Expressa uma atitude de aprovação ou de repulsa, pautada no juízo pessoal de valor, sendo observada por meio de comportamentos diversificados e relatos verbais, predominantemente afetivos ${ }^{(6)}$.

A escala de autoestima Rosenberg é um instrumento para avaliação da autoestima amplamente utilizado $^{(7-8)}$. De modo geral, a escala avalia a atitude e o sentimento positivo ou negativo por si mesmo. Níveis baixos de autoestima estão relacionados ao aparecimento de transtornos mentais como depressão, ansiedade e queixas somáticas, o que pode trazer consequências negativas na interação do binômio mãe-bebê, bem como, no desenvolvimento infantil( ${ }^{(9)}$.

Em estudo realizado com gestantes atendidas no Sistema Único de Saúde (SUS), na cidade de Pelotas, estado do Rio Grande do Sul, a média de pontos foi de 9,2 na escala de autoestima de Rosenberg, destacando-se as variáveis associadas positivamente: idade, escolaridade e condição econômica e, associação negativa, a percepção de risco à saúde do bebê e o número de gestações. Além disso, as gestantes de alto-risco apresentaram elevada autoestima quando comparadas com as de baixo-risco ${ }^{(5)}$.

No município de Juiz de Fora (MG), uma pesquisa demonstrou que mulheres mães apresentam maior média da autoestima em comparação com as não-mães. Assim, apesar da gravidez resultar em baixa média da autoestima após a criação do vínculo afetivo entre mãe e filho, a autoestima tende a melhorar ${ }^{(10)}$. Entretanto, em outro estudo, a gravidez em adolescentes resultou em redução da autoestima $^{(11)}$.
Além da autoestima, o significado de qualidade de vida durante o ciclo gravídico ainda é pouco conhecido. No entanto, existem inúmeros fatores que contribuem para um aumento da qualidade de vida, dentre eles podem ser destacados a saúde sexual que promove aprofundamento no relacionamento do casal(2); o autoconhecimento, permitindo que a gestante compreenda o momento vivido; o relacionamento familiar que propicia aportes afetivos e bem-estar(7,12).

No Brasil, em especial na região amazônica, existem poucos estudos sobre autoestima e qualidade de vida durante a gestação, sendo assim, o presente estudo visa descrever os níveis de autoestima e de qualidade de vida em uma série de gestantes atendidas na rede pública de saúde de Rio Branco, estado do Acre.

\section{MÉTODO}

Trata-se de um estudo transversal, quantitativo, realizado com gestantes no terceiro trimestre atendidas na rede pública de saúde, na cidade de Rio Branco, estado do Acre, no período de março a maio de 2011. Foi incluída na amostra mulheres com 35 semanas ou mais de gestação, idade maior ou igual de 16 anos, com companheiro, gestação de baixo risco identificada no pré-natal e residente na zona urbana.

A amostra por conveniência foi selecionada nas Unidades de Saúde (Centros de Saúde e Unidades de Saúde da Família) e na maternidade, sendo gestantes que atendiam aos critérios para participar do estudo.

O instrumento para avaliar a autoestima foi a Escala de Autoestima de Rosenberg, validada por Dini ${ }^{(13)}$. Esse instrumento é composto por dez afirmativas, cada uma com quatro opções de respostas que variam de zero a três, ou seja, concordo a discordo plenamente. Desse modo, o escore final da escala pode variar de zero (melhor autoestima) a trinta (pior autoestima).

Foi empregado para avaliar a qualidade de vida foi o WHOQOL-Bref (World Health Organization Quality of Life) proposto pela Organização Mundial de Saúde (OMS) e amplamente utilizado em diferentes países e em 20 idiomas. Disponível no Brasil, o questionário faz um inventário das atividades desenvolvidas nas duas últimas semanas e é apresentado em duas versões, a versão longa (100 questões) e a versão curta (26 questões). Esta 
última foi composta, nesta pesquisa, retirando-se das questões os melhores desempenhos psicométricos dos domínios físico, psicológico, social e ambiental ${ }^{(14)}$.

O domínio físico do WHOQOL-Bref obtém informações sobre dor e desconforto, energia e fadiga, sono e repouso, mobilidade, atividades da vida cotidiana, dependência de medicação ou de tratamentos e capacidade de trabalho. No domínio psicológico captam-se informações sobre sentimentos positivos; pensar, aprender, memória e concentração; autoestima, imagem corporal e aparência; sentimentos negativos; espiritualidade, crenças pessoais e religião. No domínio social as informações a serem obtidas referem-se às relações sociais, suporte (apoio) social e atividade sexual. Quanto ao domínio ambiental há informações sobre segurança física e proteção; ambiente no lar; recursos financeiros; cuidados de saúde e sociais: disponibilidade e qualidade; oportunidades de adquirir novas informações e habilidades; participação e/ou oportunidades de recreação e lazer; ambiente físico (poluição, ruído, trânsito e clima) e meio de transporte.

O WHOQOL-Bref é um questionário autoaplicável e o entrevistado apresenta sua resposta por meio de escores que variam de um a cinco, sendo a condição pior no escore um e a melhor no cinco. Os resultados dos domínios apresentam valores entre zero e cem, sendo piores os mais próximos de zero e melhores, os mais próximos de cem. Dessa forma, um entrevistado que apresente valor igual a 50 para determinado domínio pode ser considerado mediano para esse domínio.

As participantes também responderam questões para caracterizar o perfil socioeconômico e demográfico, dentre elas a situação conjugal foi dividida em casada (legalmente casadas) e nãocasada (união estável ou não). Vale ressaltar que as entrevistas foram realizadas em espaço reservado nas unidades de saúde, com as gestantes que cumpriam os critérios de seleção e que assinaram o Termo de Consentimento Livre e Esclarecido. O mesmo obedeceu aos dispositivos da resolução 196/96 do Conselho Nacional de Saúde e foi aprovado pelo Comitê de Ética em Pesquisa da Universidade Federal do Acre, sob o protocolo den $n^{\circ}$. 23107.017408/2010-16.

Para análise dos dados, inicialmente foram obtidas as frequências absoluta e relativa, médias e desvio-padrão das variáveis contínuas. A seguir, obtiveram-se as médias do escore de autoestima em relação às variáveis independentes (idade, cor da pele, situação conjugal, escolaridade, ocupação, rendimento familiar mensal, classificação econômica, rendimento familiar mensal, chefe da família, número de gestações, ganho de peso, gestação atual, etilismo e tabagismo) e comparadas com as médias dos escores da autoestima e da qualidade de vida, por meio do teste t Student. Também foram calculados a média e o desvio-padrão para os escores padronizados dos domínios do protocolo WHOQOL-Bref. O nível de confiança adotado na análise foi de $95 \%$. O processamento e análise dos dados foram realizados no pacote estatístico SPSS, versão 17, para o Windows.

\section{RESULTADOS}

Participaram desta pesquisa 352 gestantes, a maioria com idade entre 21 e 30 anos (66,5\%), nãobrancas, situação conjugal não-casadas, ensino médio e mais, sem ocupação e com rendimento familiar mensal de menos de dois salários mínimos, resultando em classe econômica C, De E, segundo a Associação Brasileira de Empresas de Pesquisa (ABEP) (Tabela 1).

Na maioria das residências o chefe da família era o parceiro. As gestantes eram multíparas e apresentaram ganho de peso de até $15 \mathrm{Kg}$. Das entrevistadas 25,3\% tiveram ganho de peso maior que $15 \mathrm{~kg}$ e 14,5\% afirmaram não desejar a gravidez atual. Os hábitos tabagistas e etilistas estavam presentes em $8 \%$ e 10,2\% das gestantes, respectivamente.

Ao analisar as gestantes segundo a autoestima as não-casadas e pertencentes à classe econômica $A / B$ apresentaram a menor autoestima. Nas demais variáveis analisadas não houve significância estatística, no entanto, vale mencionar que as gestantes com idade $\leq 30$ anos, brancas, com escolaridade até o ensino fundamental, sem ocupação, multíparas, com ganho de peso maior que 15 quilos, gestação atual não desejada, tabagistas e etilistas obtiveram menor autoestima (Tabela 2).

Quanto aos domínios da qualidade de vida a maior média foi obtida no Físico (75 pontos; $\pm 11,6$ ), seguido pelo social (74 pontos; $\pm 12,4$ ) e psicológico (73,3 pontos; $\pm 105,6)$. O menor escore foi observado no domínio Ambiental $(60,8$ pontos; $\pm 59,4)$, sendo este o valor com maior distanciamento dos demais domínios. Não houve diferenças nos domínios da qualidade de vida entre as categorias das variáveis independentes analisadas, exceto o domínio físico que sofreu influência em decorrência da idade das gestantes $(p>0,05)$ (Tabela 3). 
Tabela 1 - Características socioeconômicas, gestacionais e hábitos de vida da série de gestantes no terceiro trimestre. Rio Branco, 2011

\begin{tabular}{|c|c|c|}
\hline Variáveis & $\mathbf{N}$ & $\%$ \\
\hline \multicolumn{3}{|l|}{ Idade (anos) } \\
\hline 16 a 20 & 89 & 25,3 \\
\hline 21 a 25 & 144 & 40,9 \\
\hline 26 a 30 & 90 & 25,6 \\
\hline 31 a 35 & 29 & 8,2 \\
\hline \multicolumn{3}{|l|}{ Cor da pele } \\
\hline Branca & 60 & 17,1 \\
\hline Não-branca & 292 & 82,9 \\
\hline \multicolumn{3}{|c|}{ Situação conjugal } \\
\hline Casada & 73 & 20,7 \\
\hline Não casada & 279 & 79,3 \\
\hline \multicolumn{3}{|l|}{ Escolaridade } \\
\hline $\begin{array}{c}\text { Ensino } \\
\text { fundamental }\end{array}$ & 117 & 33,3 \\
\hline $\begin{array}{l}\text { Ensino médio/ } \\
\text { superior }\end{array}$ & 235 & 66,7 \\
\hline \multicolumn{3}{|l|}{ Ocupação } \\
\hline Sim & 111 & 31,5 \\
\hline Não & 241 & 68,5 \\
\hline \multicolumn{3}{|c|}{ Renda familiar mensal em salários mínimos $(\mathrm{SM})(\mathrm{N}=349)$} \\
\hline$<$ dois SM & 210 & 60,2 \\
\hline$\geq$ dois SM & 139 & 39,8 \\
\hline \multicolumn{3}{|c|}{ Classificação Econômica } \\
\hline $\mathrm{A} / \mathrm{B}$ & 29 & 8,2 \\
\hline $\mathrm{C} / \mathrm{D} / \mathrm{E}$ & 323 & 91,8 \\
\hline \multicolumn{3}{|l|}{ Chefe da família } \\
\hline Parceiro & 309 & 87,8 \\
\hline Outro & 43 & 12,2 \\
\hline \multicolumn{3}{|c|}{ Número de gestações } \\
\hline Primigesta & 143 & 40,6 \\
\hline Duas ou mais & 209 & 59,4 \\
\hline \multicolumn{3}{|c|}{ Ganho de peso em quilogramas } \\
\hline Até 15 & 263 & 74,7 \\
\hline De 16 a mais & 89 & 25,3 \\
\hline \multicolumn{3}{|l|}{ Gestação Atual } \\
\hline Desejada & 301 & 85,5 \\
\hline Não desejada & 51 & 14,5 \\
\hline \multicolumn{3}{|l|}{ Tabagista } \\
\hline Sim & 28 & 8 \\
\hline Não & 324 & 92 \\
\hline \multicolumn{3}{|l|}{ Etilista } \\
\hline Sim & 36 & 10,2 \\
\hline Não & 316 & 89,8 \\
\hline Total & 352 & 100 \\
\hline
\end{tabular}

Tabela 2 - Média e desvio-padrão da Autoestima segundo variáveis de uma série de gestantes. Rio Branco, 2011

\begin{tabular}{|c|c|c|c|}
\hline \multirow{2}{*}{ Variáveis } & \multicolumn{3}{|c|}{ Autoestima (Escala de Rosenberg) } \\
\hline & $x$ & $\mathrm{dp}$ & $\mathrm{p}$ \\
\hline \multicolumn{3}{|l|}{ Idade (anos) } & \multirow{3}{*}{0,181} \\
\hline$\leq 30$ & 8,65 & 3,96 & \\
\hline$>30$ & 7,59 & 4,98 & \\
\hline \multicolumn{3}{|l|}{ Cor da pele } & \multirow{3}{*}{0,342} \\
\hline Branca & 9,02 & 4,07 & \\
\hline Não-branca & 8,47 & 4,06 & \\
\hline \multicolumn{3}{|c|}{ Situação conjugal } & \multirow{3}{*}{$<0,001$} \\
\hline Casada & 7,21 & 4,03 & \\
\hline Não-casada & 8,92 & 4,06 & \\
\hline \multicolumn{3}{|l|}{ Escolaridade } & \multirow{3}{*}{0,152} \\
\hline $\begin{array}{l}\text { Ensino } \\
\text { fundamental }\end{array}$ & 9,01 & 4,06 & \\
\hline $\begin{array}{l}\text { Ensino } \\
\text { médio/ superior }\end{array}$ & 8,34 & 4,04 & \\
\hline \multicolumn{3}{|l|}{ Ocupação } & \multirow{3}{*}{0,303} \\
\hline Sim & 8,23 & 4,04 & \\
\hline Não & 8,71 & 4,06 & \\
\hline
\end{tabular}

Renda familiar mensal em salários mínimos (SM)

\begin{tabular}{lll}
$<$ dois SM & 8,76 & 4,06 \\
\hline$\geq$ dois SM & 8,51 & 4,04
\end{tabular}$\quad 0,564$

Classificação Econômica

\begin{tabular}{|c|c|c|c|}
\hline $\mathrm{A} / \mathrm{B}$ & 8,67 & 4,06 & \multirow[t]{2}{*}{0,050} \\
\hline $\mathrm{C} / \mathrm{D} / \mathrm{E}$ & 7,34 & 3,97 & \\
\hline \multicolumn{3}{|c|}{ Chefe da família } & \multirow{3}{*}{0,931} \\
\hline Parceiro & 8,57 & 4,06 & \\
\hline Outro & 8,51 & 4,05 & \\
\hline
\end{tabular}

Número de gestações

$\begin{array}{lll}\text { Primigesta } & 8,33 & 4,08 \\ \text { Duas ou mais } & 8,72 & 4,04\end{array} \quad 0,372$

Ganho de peso em quilogramas

\begin{tabular}{|c|c|c|c|}
\hline Até 15 & 8,45 & 4,06 & \multirow[t]{2}{*}{0,375} \\
\hline De 16 a mais & 8,90 & 4,03 & \\
\hline \multicolumn{3}{|l|}{ Gestação Atual } & \multirow{3}{*}{0,404} \\
\hline Desejada & 8,12 & 4,00 & \\
\hline Não desejada & 8,64 & 4,06 & \\
\hline \multicolumn{3}{|l|}{ Tabagista } & \multirow{3}{*}{0,912} \\
\hline Sim & 8,64 & 4,07 & \\
\hline Não & 8,55 & 4,04 & \\
\hline \multicolumn{3}{|l|}{ Etilista } & \multirow{3}{*}{0,210} \\
\hline Sim & 9,36 & 4,07 & \\
\hline Não & 8,47 & 4,04 & \\
\hline Total & 8,56 & 4,07 & \\
\hline
\end{tabular}


Tabela 3 - Qualidade de vida, segundo média e desvio-padrão das variáveis independentes, da série de gestantes. Rio Branco, 2011

\begin{tabular}{|c|c|c|c|c|c|c|c|c|c|c|c|c|}
\hline \multirow{3}{*}{ Variáveis } & \multicolumn{12}{|c|}{ Qualidade de Vida (WHOQOL-Bref) } \\
\hline & \multicolumn{3}{|c|}{ Físico } & \multicolumn{3}{|c|}{ Psicológico } & \multicolumn{3}{|c|}{ Social } & \multicolumn{3}{|c|}{ Ambiental } \\
\hline & $\mathbf{x}$ & $\Sigma$ & p & $x$ & $\sigma$ & $\mathbf{p}$ & $\mathbf{x}$ & $\sigma$ & $\mathbf{p}$ & $x$ & $\bar{\Sigma}$ & $\mathbf{P}$ \\
\hline \multicolumn{13}{|l|}{ Idade (anos) } \\
\hline$\leq 30$ & 75,4 & 11,68 & \multirow{2}{*}{0,044} & 73,4 & 10,49 & \multirow{2}{*}{0,446} & 74,9 & 12,65 & \multirow{2}{*}{$-0,503$} & 61,1 & 10,34 & \multirow{2}{*}{0,139} \\
\hline$>30$ & 70,8 & 10,33 & & 71,8 & 10,19 & & 73,3 & 10,05 & & 58,1 & 11,16 & \\
\hline \multicolumn{13}{|l|}{ Cor da pele } \\
\hline Branca & 75,5 & 12,59 & \multirow{2}{*}{0,717} & 74,0 & 10,78 & \multirow{2}{*}{0,571} & 73,9 & 13,24 & \multirow{2}{*}{$-0,551$} & 61,0 & 11,46 & \multirow{2}{*}{0,864} \\
\hline Não-branca & 74,9 & 11,45 & & 73,1 & 10,41 & & 74,9 & 12,30 & & 60,8 & 10,22 & \\
\hline \multicolumn{13}{|c|}{ Situação conjugal } \\
\hline Casada & 74,6 & 10,04 & \multirow{2}{*}{0,760} & 71,9 & 8,41 & \multirow{2}{*}{0,200} & 75,1 & 9,37 & \multirow{2}{*}{0,787} & 61,4 & 9,13 & \multirow{2}{*}{0,610} \\
\hline Não-casada & 75,1 & 12,03 & & 73,6 & 10,92 & & 74,7 & 13,15 & & 60,7 & 10,75 & \\
\hline Escolaridade & & & & & & & & & & & & \\
\hline $\begin{array}{l}\text { Ens. } \\
\text { fundamental }\end{array}$ & 73,9 & 12,15 & & 72,9 & 11,60 & & 73,7 & 12,93 & & 59,9 & 11,20 & \\
\hline $\begin{array}{l}\text { Ens. médio/ } \\
\text { superior }\end{array}$ & 75,5 & 11,35 & & 73,4 & 9,87 & & 75,3 & 12,20 & & 61,3 & 10,01 & , \\
\hline Ocupação & & & & & & & & & & & & \\
\hline Sim & 75,1 & 12,35 & & 73,5 & 10,43 & 0777 & 75,3 & 12,61 & 0584 & 60,9 & 11,23 & 0.910 \\
\hline Não & 74,9 & 11,31 & 0 & 73,2 & 10,50 & 172 & 74,5 & 12,40 & , & 60,8 & 10,06 & (1) \\
\hline Renda familiar & mensa & em salá & rios mín & $\operatorname{mos}(\mathrm{S}$ & & & & & & & & \\
\hline$<$ dois SM & 75,6 & 10,76 & קר? & 73,3 & 10,93 & 0832 & 75,0 & 13,52 & 0605 & 60,6 & 10,90 & 0.875 \\
\hline$\geq$ dois SM & 74,0 & 12,23 & $\angle U L$ & 73,0 & 9,68 & 0,035 & 74,3 & 10,80 & 0,005 & 60,8 & 9,31 &, 0 \\
\hline Classificação e & conôm & & & & & & & & & & & \\
\hline $\mathrm{A} / \mathrm{B}$ & 75,9 & 11,52 & 670 & 72,0 & 11,02 & 0402 & 74,7 & 13,99 & 0987 & 62,6 & 11,86 & 0230 \\
\hline $\mathrm{C} / \mathrm{D} / \mathrm{E}$ & 74,9 & 11,66 & $0 / 0$ & 73,4 & 10,42 & $0,4 J 5$ & 74,8 & 12,33 & 0,302 & 60,7 & 10,29 & צרנד, \\
\hline Chefe da famíli & & & & & & & & & & & & \\
\hline Parceiro & 72,0 & 17,97 & 224 & 71,8 & 12,85 & 320 & 76,7 & 16,42 & 280 & 61,2 & 12,41 & 800 \\
\hline Outro & 75,4 & 10,43 & 84 & 73,5 & 10,09 & ט, & 74,5 & 11,80 & 0,500 & 60,8 & 10,14 & , \\
\hline Número de ges & stações & & & & & & & & & & & \\
\hline Primigesta & 74,9 & 12,74 & 42 & 72,6 & 10,15 & 315 & 75,4 & 13,96 & 27 & 60,9 & 10,30 & 2 \\
\hline 2 ou mais & 75,0 & 10,84 & & 73,7 & 10,67 & נוכנס & 74,3 & 11,32 & +22 & 60,8 & 10,53 & 002 \\
\hline Ganho de peso & eem qu & ograma: & & & & & & & & & & \\
\hline Até 15 & 75,8 & 12,34 & 64 & 73,8 & 12,60 & 33 & 75,7 & 13,77 & 435 & 61,9 & 11,43 & 2 \\
\hline 16 ou mais & 74,7 & 11,40 & & 73,1 & 9,66 & S, & 74,5 & 11,98 & & 60,5 & 10,06 & 202 \\
\hline Gestação atual & & & & & & & & & & & & \\
\hline Desejada & 75,3 & 10,58 & & 73,4 & 9,64 & & 75,3 & 11,36 & & 60,7 & 10,24 & \\
\hline Não desejada & 73,2 & 16,57 & 84 & 72,1 & 14,49 & 0,536 & 71,7 & 17,41 & 166 & 61,7 & 11,52 & , \\
\hline Tabagista & & & & & & & & & & & & \\
\hline Sim & 74,9 & 14,95 & & 74,9 & 12,29 & & 76,8 & 14,93 & & 60,8 & 10,81 & \\
\hline Não & 75,0 & 11,33 & & 73,1 & 10,30 & 0,402 & 74,6 & 12,23 & $0,3 / 1$ & 60,8 & 10,41 & , \\
\hline Etilista & & & & & & & & & & & & \\
\hline Sim & 71,9 & 21,40 & & 70,0 & 15,62 & & 76,6 & 20,68 & & 62,7 & 14,54 & \\
\hline Não & 75,3 & 9,94 & & 73,6 & 9,67 & 0,105 & 74,6 & 11,17 & 0,359 & 60,6 & 9,86 & \\
\hline Total & 75,0 & 11,6 & & 73,3 & 10,5 & & 74,8 & 12,4 & & 60,8 & 59,4 & \\
\hline
\end{tabular}




\section{DISCUSSÃO}

A gestação representa um período único e especial na vida da mulher, no qual ao assumir a identidade de tornar-se mãe os sentimentos podem alternar entre alegria e incertezas, medos e segurança ou inseguranças ${ }^{(15)}$.

Desde as crenças antigas, a gestação se encontra vinculada à figura feminina(16), provocando a redefinição de seus valores, estima e identidade ${ }^{(12)}$, exigindo da gestante adaptações constantes em razão das transformações internas e externas ${ }^{(17)}$ que ocorrem durante o período gestacional.

A autoestima é uma das temáticas da avaliação psicológica da gestante que é pouco estudada ${ }^{(9)}$. A elevada autoestima da mãe é considerada um fator de proteção para o desenvolvimento infantil e para a depressão durante e após a gravidez ${ }^{(18-19)}$.

A idade é um fator de risco para baixa autoestima em gestantes, tendo sido observada em adolescentes ${ }^{(20-21)}$. Em pesquisa realizada na cidade de Pelotas, Rio Grande do Sul, verificou-se que as mulheres até 18 anos, sem companheiro, com baixa escolaridade, gestação atual não planejada, multíparas e que usaram algum tipo de droga apresentaram menor autoestima ${ }^{(5)}$. No presente estudo apesar de não termos uma diferenciação quanto ao grupo de adolescentes cita-se a semelhança nos achados entre as idades com 30 anos ou menos.

A média da autoestima das gestantes de Pelotas, Rio Grande do Sul, foi de 9,3 pontos $^{(9)}$, valor maior que o encontrado nas gestantes de Rio Branco, demonstrando que estas tinham maior autoestima.

A situação conjugal durante a gestação mostrou diferença significativa quanto à autoestima, sendo a pior autoestima encontrada nas mulheres nãocasadas, fato este que pode estar associado a segurança obtida com o casamento tradicional ${ }^{(22)}$. O parceiro é a fonte de apoio e segurança para a gestante sendo capaz de aumentar sua autoestima, por meio da atenção e carinho durante a gravidez e parto, sendo também responsável por auxiliar no controle e redução da sensação de dor. A participação do homem em todo o processo é nova, e resulta da luta pela humanização da assistência à gestante ${ }^{(23)}$. Vale mencionar que a falta de apoio do parceiro para cuidar do bebê também foi associada à baixa autoestima em grávidas ${ }^{(24)}$. Em estudo realizado em uma Unidade de Saúde da Família de Recife, estado de Pernambuco, evidenciou que há baixo envolvimento paterno no período pré-natal ${ }^{(25)}$.

Em estudo para avaliar os fatores associados a sintomas de depressão em gestantes observouse que a situação conjugal instável ou ausência de um parceiro, a falta de apoio social e o não planejamento da gravidez foram considerados fatores de risco para o aparecimento desses sintomas ${ }^{(26)}$.

As gestantes pesquisadas classificadas segundo a condição socioeconômica em A/B obtiveram uma menor autoestima, fato que pode estar associado com as mudanças físicas e comportamentais relacionadas com a gravidez. No entanto não foi encontrado na literatura estudos para corroborar com esse achado.

A gravidez, por meio de suas alterações fisiológicas, pode provocar alterações no modo como a mulher percebe a sua qualidade de vida e a sua saúde ${ }^{(27)}$. Deste modo, o pré-natal é uma ferramenta importante para a manutenção da qualidade de vida durante a gravidez por permitir um olhar integral e ampliado a mulher gestante $^{(8)}$. As características que cada mulher apresenta individualmente e as que estão relacionadas a condições sociodemográficas podem interferir no desenvolvimento de uma gestação saudável, consequentemente alterando a sua qualidade de vida $^{(7,28)}$. As gestantes pesquisadas obtiveram diferença significativa neste domínio em decorrência da idade.

Em estudo desenvolvido com 120 gestantes no município de Souza, no estado da Paraíba, na qualidade de vida, quanto ao domínio físico, incluíram as insatisfações: dor; desconforto; fadiga; alterações no sono e repouso e falta de energia. No domínio psicológico ressaltam-se alterações em imagem corporal e aparência, memória, concentração e sentimentos negativos; no domínio social a insatisfação esteve relacionada com a atividade sexual; no ambiental a maior insatisfação foi mencionada quanto aos recursos financeiros, oportunidade de lazer e transporte ${ }^{(29)}$.

Na série de gestantes da presente investigação os domínios com menores escores de qualidade de vida foram para os domínios ambiental e psicológico. Em estudo realizado com 42 gestantes de uma Unidade de Saúde da Família da periferia de São Bernardo do Campo foram verificados escores médios de 57,65 para o 
domínio físico, 68,75 para o psicológico, 59,75 para o ambiental e 77,98 para o domínio social da qualidade de vida(8), sendo observados valores próximos aos encontrados na presente investigação para os domínios ambiental e social.

$\mathrm{Na}$ investigação com um grupo de gestantes diabéticas e não diabéticas, realizado em Botucatu, estado de São Paulo, foi observado que tanto as diabéticas como as normoglicêmicas no final da gravidez apresentaram menores escores para todos os domínios da qualidade de vida em comparação às gestantes da presente investigação, exceto no domínio ambiental ${ }^{(30)}$. Já em outra pesquisa sobre exercício aquático e qualidade de vida, conduzida na cidade de Campinas, também no estado de São Paulo, mulheres na $36^{a}$ semana de gestação, apresentaram cerca de 67 pontos no domínio físico e 60 pontos no domínio ambiental, sendo a média geral entorno dos 70 pontos $^{(31)}$, demonstrando, no que tange ao domínio ambiental, similaridade com o observado entre as grávidas do presente estudo, com a qualidade de vida melhor percebida nos demais domínios.

Um ensaio clínico conduzido no Centro de Atendimento Integral à Saúde da Mulher da Universidade Federal de Campinas, também sobre exercícios físicos e qualidade de vida, demonstrou que no final da gestação todos os domínios da qualidade de vida ficaram abaixo de 70 pontos, com menor escore entre o grupo de não praticantes de exercícios físicos, 42 e 55 pontos respectivamente nos domínios físico e ambiental ${ }^{(32)}$.

Conclui-se, então, que há uma tendência nesta pesquisa e em outras investigadas que o domínio ambiental tem obtido os menores escores entre gestantes quando se trata de qualidade de vida e está relacionada a alguma ou algumas das seguintes variáveis: segurança física e proteção; ambiente no lar; recursos financeiros; cuidados de saúde e sociais: disponibilidade e qualidade; oportunidades de adquirir novas informações e habilidades; participação e/ou oportunidades de recreação e lazer; ambiente físico (poluição, ruído, trânsito e clima) e meio de transporte.

Uma limitação da presente pesquisa é o fato dos dados não poderem ser generalizados a todas as gestantes por ser uma amostra por conveniência. No entanto, permitem a compreensão de aspectos individuais e a exposição de variáveis de maior fragilidade durante o período gestacional. Vale mencionar que a pesquisa, mesmo com um número reduzido de gestantes, permitiu a abrangência de todas as Unidades Básicas da cidade de Rio Branco, estado do Acre.

\section{CONCLUSÃO}

Verificou-se neste estudo que a autoestima das gestantes não-casadas e classificadas segundo a condição econômica em A e B apresentaram a menor autoestima. Além disso, os menores escores da autoestima foram observados nas idades mais jovens, com baixa escolaridade, sem ocupação, nas com ganho de peso maior que 15 quilos, com gestações anteriores, gravidez atual não desejada, etilistas e tabagistas. Quanto à qualidade de vida os menores escores da qualidade de vida foram obtidos no domínio psicológico e ambiental, tendo o domínio físico apresentado diferença para as faixas etárias analisadas.

Cabe ressaltar que o presente estudo deve ser visto como uma abordagem inicial à temática das consequências da maternidade para a autoestima e qualidade de vida feminina. Nesse sentido, seria interessante a realização de futuras investigações envolvessem maiores amostras populacionais, visto que o aprofundamento da compreensão da relação existente entre gravidez e autoestima/ qualidade de vida é uma necessidade para a manutenção da saúde da gestante e do feto.

\section{REFERÊNCIAS}

1. Costa GRC, Chein MBC, Gama MEA, Coelho LSC, Costa ASV, Cunha CLF et al. Caracterização da cobertura do pré-natal no Estado do Maranhão, Brasil. Rev Bras Enferm 2010; 63(6):1005-9. doi: http://dx.doi. org/10.1590/S0034-71672010000600021

2. Vido MB. Qualidade de vida na gravidez [dissertação]. São Paulo: Universidade de Guarulhos; 2006. p.98.

3. Camacho KG, Vargens OMC, Progianti JM. Adaptandose à nova realidade: a mulher grávida e o exercício de sua sexualidade. Rev. enferm. UERJ 2010; 18:32-37.

4. Falcone VM, Mader CVN, Nascimento CFL,Santos JMM, Nóbrega FJ. Atuação multiprofissional e a saúde mental de gestantes. Rev Saúde Pública. 2008; 39(4):612-18. doi: http://dx.doi.org/10.1590/S003489102005000400015

5. Dias M, Silva RA, Souza LD, Lima RC, Pinheiro RT, Moraes IG. Self-esteem and associated factors in 
pregnant women in the city of Pelotas, Rio Grande do Sul State, Brazil. Cad Saúde Pública. 2008; 24(12):2787-97. doi: http://dx.doi.org/10.1590/S0102311X2008001200007

6. Rosenberg M. Society and the adolescent self-image. Princeton, NJ: Princeton University Press; 1965.

7. Avanci JQ, Assis SG, Santos NC, Oliveira RVC. Adaptação transcultural de escala de autoestima para adolescentes. Psicol. reflex. crit. 2006;20(3):397405. doi: http://dx.doi.org/10.1590/S010279722007000300007

8. Castro DFA, Fracolli LA. Qualidade de vida e promoção da saúde: em foco as gestantes. O Mundo da Saúde 2013; 37(2):159-65.

9. Silva RA, Ores LC, Mondin TC, Rizzo RN, Moraes IGS, Jansen $\mathrm{K}$ et al. Transtornos mentais comuns e autoestima na gestação: prevalência e fatores associados. Cad. saúde pública 2010; 26(9):1832-8. doi: http://dx.doi.org/10.1590/S0102-311X2010000900016

10. Souza D, Ferreira MC. Auto-estima pessoal e coletiva em mães e não-mães. Psicol Estud. 2005; 10(1):19-25.

11. Rodrigues JE, Nascimento MV, Filho AVD, PontesBarros JF, Magalhães MN, Gomes CAFP et al. Avaliação da autoestima de adolescentes gestantes de baixa renda assistidas pelo Núcleo de Apoio à Saúde da Família. Conscientiae saúde 2010; 9(3):395-401.

12. Brasil. Ministério da Saúde. Manual técnico de prénatal e puerpério, atenção qualificada e humanizada. Brasília: Ministério da Saúde; 2012.

13. Dini GM. Adaptação cultural e validação da versão brasileira da escala de auto-estima de Rosenberg. Rev. Soc. Bras. Cir. Plás. 2004; 19(1):41-52.

14. Fleck MP, Louzada S, Xavier M, Chachamovich E, Vieira G, Santos L et al. Aplicação da versão em português do instrumento abreviado de avaliação da qualidade de vida "WHOQOL-bref". Rev de Saúde Pública 2000; (34):178-183. doi: http://dx.doi. org/10.1590/S0034-89102000000200012

15. Tedesco RP, Filho NLM, Mathias L, Benez AL, Castro VCL, Bourroul GM et al. Fatores determinantes para as expectativas de primigestas acerca da via de parto. Rev. bras. ginecol. obstet. 2004; 26(10):791-798. doi: http://dx.doi.org/10.1590/S0100-72032004001000006

16. Moura, R. Iconografias do feminino: Mitos, arte e outras representações. História, imagem e narrativas. 2010; 10(1): 1-12.

17. Camacho KG, Vargens OMC, Progianti JM, Spíndola T. Vivenciando repercussões e transformações de uma gestação: perspectivas de gestantes. 2010; Cienc. enferm. 16(2): 115-125.
18. Maia JMD, Williams B, Albuquerque LC. Fatores de risco e fatores de proteção ao desenvolvimento infantil: uma revisão da área. Temas psicol. 2005; 13(2):91-103.

19. Hudson DB, Elek SM, Campbell-Grossman C. Depression, self-esteem, loneliness, and social support among adolescent mothers participating in the New Parents Project. Adolescence 2000; 35(139):445-53.

20. Cabral FB, Ressel LB, Landerdahl MC. Consulta de enfermagem: estratégia de abordagem à gestante na perspectiva de gênero. Esc. Anna Nery 2005; 9(3):459-65. doi: http://dx.doi.org/10.1590/S141481452005000300014

21. Gomes KRO, Speizer IS. Longitudinal Study on Self-Esteem among Recently Pregnant Brazilian Adolescents. Journal of Reproductive and Infant Psychology. 2010; 28(4): 359-71.

22. Zordan EP, Falcke D, Wagner A. Casar ou não casar? Motivos e expectativas com relação ao casamento. Psicol. Rev. 2009; 15(2): 56-76.

23. Junckes JM, Guesser JC, Zampieri MFM, Gregório VRP, Oliveira ZC, Regis I. Grupo de gestantes e/ou casais grávidos e a inserção do acompanhante/pai no processo de nascimento. Extensio: R. Eletr. de Extensão 2009;6(7):55-72. doi: http://dx.doi.org/10.5007/1807-0221.2009v6n7p55

24. Maçola L, Vale IN, Carmona EV. Assessment of selfesteem in pregnant women using Rosenberg's SelfEsteem Scale. Rev. esc. enferm. USP. 2010; 44(3):570-77.

25. Oliveira SC, Ferreira JG, Silva PMP, Ferreira JM, SeabraRA, Fernando VCN. A participação do homem/ pai no acompanhamento da assistência pré-natal. Cogitare Enferm. 2009; 14(1):73-8. doi: http://dx.doi. org/10.5380/ce.v14i1.14118

26. Nascimento SRC, Amorim MHC, Primo CC, Castro DS. Fatores de risco para o desenvolvimento de depressão na gestação. Rev. Bras. Pesqui. Saude 2009; 11(2):18-23.

27. Pimentel MO. Qualidade de vida relacionada à saúde de mulheres grávidas com baixo nível socioeconômico, em São Paulo/SP [dissertação]. São Paulo (SP): Escola de Enfermagem, Universidade de São Paulo; 2006. 86 p.

28. Afonso A, Quitério D, Sousa F, Sousa L, Silva R, Henrique $\mathrm{CMG}$, et al. A influência dos factores demográficos, sociais, económicos e obstétricos na qualidade de vida da grávida. INFAD Revista de Psicología. 2011, 4(1): 39-44.

29. Oliveira SX, Oliveira MB, Silva RAR, Davim RMB. Características dos domínios da qualidade de vida em gestantes da estratégia saúde da família. Rev. Enferm. UFPE 2013; 7(esp):7007-16. doi: 10.5205/reuol.476742136-1-ED.0712esp201307

30. Marquesim NAQ. Qualidade de vida, ansiedade 
e depressão em gestantes diabéticas ou com hiperglicemia leve. [dissertação]. Botucatu (SP): Universidade Estadual Paulista, Faculdade de Medicina de Botucatu; 2013. 66 p.

31. Vallim AL, Osis MJ, Cecatti JG, Baciuk EP, Silveira C1, Cavalcante SR. Water exercises and quality of life during pregnancy. Reprod Health, 2011; 8:14. doi:10.1186/1742-4755-8-14

32. Nascimento SL, Surita FG, Parpinelli MA, Siani S, Pinto e Silva JL. The effect of an antenatal physical exercise programme on maternal/perinatal outcomes and quality of life in overweight and obese pregnant women: a randomised clinical trial. BJOG, 2011;(118):1455-63. doi: 10.1111/j.1471-0528.2011.03084.x 